\title{
Bilateral tuberculous orchitis; a case report
}

\author{
Wijayagunawardane S, Gihan LU, De Silva C, Abeygunasekera AM \\ Urology Unit, Colombo South Teaching Hospital, Kalubowila
}

Corresponding author: Dr.A.M.Abeygunasekera(amabey@sltnet.lk)

\section{Introduction}

Although tuberculosis (TB) was known to have existed for more than 7000 years the term genitourinary tuberculosis (GUTB) was coined only in 1937. It is credited to Wildbolz who showed that renal and epididymal TB were local manifestations of the same blood borne infection. GUTB is the second most common form of extra-pulmonary TB in most countries. But according to the figures of the National Programme for Tuberculosis Control and Chest Diseases, the reported rate of GUTB is very low in Sri Lanka (1) with only four cases of GUTB recorded in 2007.

\section{Case Report}

A 48-year old man noticed his right testis to be enlarged. As the enlargement increased slowly over the next 4 months he sought medical advice. There was no pain. He had undergone left hydrocelectomy four years ago. He had no urinary symptoms. On examination, both testes were enlarged and firm in consistency. The outline was regular and the surface was smooth (Fig 1). There was no tenderness. There were no other clinically detectable abnormalities.

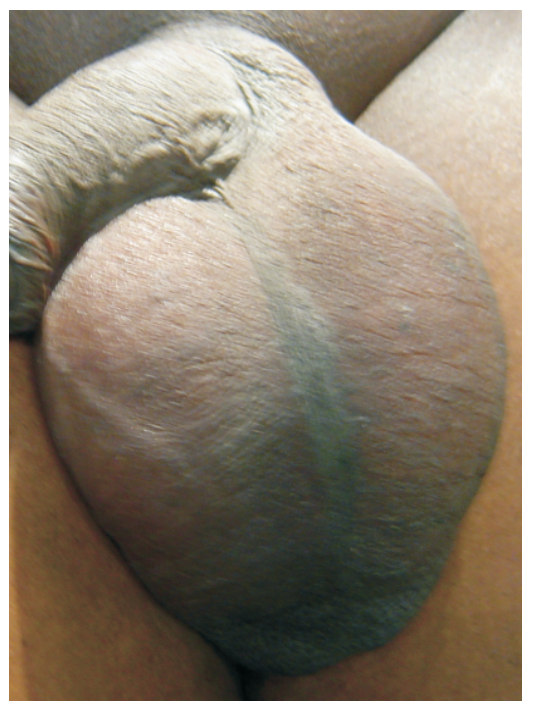

Figure 1 - External appearance of the genitalia
An ultrasonography showed both testes to contain lesions suggestive of tumours and the lesion in the left testis was larger. There was no para-aortic lymphadenopathy. Serum alpha fetoprotein and beta HCG (human chorionic gonadotrophin) levels were normal. The Mantoux test was negative and the ESR was $28 \mathrm{~mm}$ in the first hour. Left inguinal orchiectomy was done as the lesion on the left testis was bigger. The cut surface of the specimen revealed caseous lesions in the left testis and epididymis (Fig 2). The histology revealed granulomatous lesions in the testis diagnostic of tuberculous orchitis.

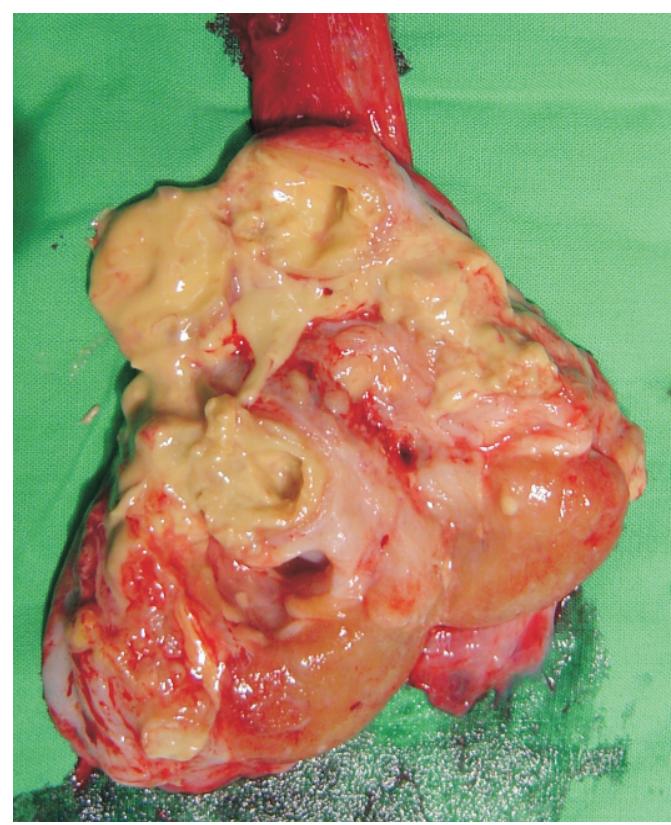

Figure 2 - Cut section of the left testicle

\section{Discussion}

Epididymal TB is a common form of GUTB. However involvement of the testis is rare and occurs in about $10 \%$ of cases of epididymal TB. Bilateral tuberculous orchitis is very rare. Generally the spread of the Mycobacterium tuberculosis to the epididymis occurs via blood but occasional reports of possible direct or lymphatic spread from lower 
urinary tract have been reported (2). The organism enters the testis from the epididymis by direct invasion. Most patients with epididymal TB have no evidence of renal TB. Almost all patients with epididymal and testicular TB do not have urinary symptoms.

GUTB is common in the 20-50 years age group and not in the old, frail patients. Diagnosis in the initial stage of the disease is difficult. Painful enlargement of the testis and epididymis simulates bacterial orchitis. Later the swelling becomes nodular, firm and painless. Most of the time, M. tuberculosis cannot be isolated from urine. Presence of a sinus in the scrotum suggests the diagnosis. Otherwise biopsy of the epididymis or orchiectomy is necessary to confirm the diagnosis before commencing treatment. Fine needle aspiration cytology of the epididymal mass may suggest the diagnosis in some. The clinical features may simulate a testicular tumour. About $10 \%$ of patients with tuberculous orchitis may undergo inguinal (high) orchiectomy for a suspected tumour (3). Though Mantoux test is useful in the diagnosis of renal TB, it is normal in most patients with epididymal TB. Same is true for ESR.
Epididymal and testicular $\mathrm{TB}$ can be treated effectively with the standard six-month regime of anti-tubercular therapy. Few patients with a persistent scrotal sinus my require epididymectomy later. The transmission of genital TB from male to female is very rare. Occasional reports of pelvic tuberculosis in the sexual partner of patients with tuberculous epididymo-orchitis suggest the possibility of female to male transmission.

\section{References}

1. Annual report of the National Programme for Tuberculosis Control and Chest Diseases 2007 Ministry of Healthcare \& Nutrition, Sri Lanka.

2. Cek M, Lenk S, Naber KG, Bishop MC et al. EAU guidelines for the management of genitourinary tuberculosis. European Urology 2005; 48: 353-62

3. Viswaroop BS, Kekre N, Gopalakrishnan G. A study of isolated epididymal tuberculosis. Journal of Postgraduate Medicine 2005; 81: 109-11.

\title{
A girl with complicated Stevens Johnson syndrome following ciprofloxacin; a case report
}

\author{
Nanayakkara PGCJ, Lekamwasam S, Pratheepan GJ \\ University Medical Unit, Teaching Hospital, Karapitiya, Galle \\ Corresponding author; Dr.P.G.C.J.Nanayakkara (chathu.nanayakkara@yahoo.com)
}

\section{Introduction}

Stevens Johnson syndrome (SJS) is a multi-systemic disorder with predominant dermatological manifestations. We report a patient who had multiorgan involvement with life threatening complications.

\section{Case Report}

An eighteen year old, previously healthy girl presented to a General Practitioner with fever, dysuria and right sided loin pain of three days duration. After urinanalysis, she has been started on oral ciprofloxacin. About ten hours after the first dose, she noticed a painful skin rash over the body, lips and vulva and felt gritty sensation in the eyes.

On admission, she was febrile $\left(102^{\circ} \mathrm{F}\right)$. Diffuse mucosal ulcerations in conjunctiva, oral cavity and vulva were seen while vesiculo-bullous and targetoid eruptions were detected in the body and limbs. Furthermore, she was detected to have severe membranous conjunctivitis with epithelial defects and conjunctival dryness, favoring an allergic reaction. The clinical diagnosis of SJS was made and supportive care was initiated. 